\title{
Ligadura endoscópica como tratamiento de la enfermedad hemorroidal
}

\author{
Homero Rodríguez-Zentner, Emmy Arrue, Moisés Cukier \\ Departamento de Cirugía, División de Cirugía. Pacífica Salud - Hospital \\ Punta Pacífica, Panamá, Panamá
}

\begin{abstract}
RESUMEN
Introducción: Entre los procedimientos para el tratamiento de las hemorroides, la ligadura con banda es el más utilizado. Aportamos nuestra experiencia realizando este procedimiento con la ayuda visual del colonoscopio y el 6 Shooter ${ }^{\circledR}$ Universal Saeed® Multi-Band Ligator.

Objetivo: Analizar los beneficios de la ligadura endoscópica de hemorroides internas que no han respondido al tratamiento convencional.

Diseño: Se trata de un estudio observacional descriptivo, retrospectivo de revisión de expedientes.

Material y métodos: Se incluyeron los pacientes con hemorroides sintomáticos entre los grados I a III sin respuesta a tratamiento convencional a los cuales se les realizó ligadura endoscópica de las mismas entre febrero de 2015 y febrero 2020. En todos los casos, el procedimiento se realizó en la suite de endoscopía, con el paciente sedado y monitorizado por un anestesiólogo.

Resultados: Se efectuó el procedimiento en 146 pacientes, 92 varones. La edad media fue 44,0 años (21-78 DE $\pm 11,23)$. El tiempo medio del procedimiento fue 12,38 minutos (6-19 DE $\pm 2,98)$. A las 24 horas, en una escala del 1-10, los pacientes describieron el dolor en una media de 4, a los 7 días en 1,5 y a los 30 días en cero. Tres pacientes presentaron retención aguda de orina y 3 pacientes trombosis externa. Ningún paciente presentó fiebre, sepsis o perforación durante la retroflexión.

Conclusión: La ligadura endoscópica de hemorroides es una técnica reproducible, segura, que permite el tratamiento de hemorroides sintomáticas grados I a III en su gran mayoría, en una sola sesión.
\end{abstract}

Palabras claves: Enfermedad hemorroidal; Endoscopía; Ligadura

\begin{abstract}
Introduction: Among the procedures for the treatment of hemorrhoids, band ligation is the most commonly used. In this study we present our experience performing this procedure with the visual aid of the colonoscope and the 6 Shooter ${ }^{\circledR}$ Universal Saeed® Multi-Band Ligator.

Objective: To analyze the benefits of endoscopic ligation of internal hemorrhoids unresponsive to conventional treatment. Design: Retrospective, descriptive, observational study.

Material and methods: The records of all patients with symptomatic grade I to III hemorrhoids without response to conventional treatment were reviewed. Those who underwent ligation between February 2015 and February 2020 were included. In all cases the procedure was performed in the endoscopy suite with the patient sedated and monitored by an anesthesiologist.

Results: One hundred-forty-six patients (92 men) underwent the procedure. The mean age was $44 \pm 11.23$ (21-78) years. The mean duration of the procedure was $12.38 \pm 2.98$ (6-19) minutes. On a scale of 1-10, patients gave the pain symptom an average score of 4 at 24 hours, 1.5 at 7 days, and 0 at 30 days. Three patients had acute urine retention and 3 had external thrombosis. No patient had fever, sepsis, or perforation.

Conclusion: Endoscopic ligation is a safe and reproducible technique that allows the treatment of symptomatic grade I to III hemorrhoids usually in a single session..
\end{abstract}

Key words: Hemorrhoidal Disease; Endoscopy; Ligation

\section{INTRODUCCIÓN}

Las afecciones relacionadas a las hemorroides, son de las causas más frecuentes de consulta coloproctológica. ${ }^{1} \mathrm{La}$ presentación clínica más frecuente se caracteriza por sangrado indoloro con la defecación que puede estar acompañado o no de prolapso del tejido hemorroidal. ${ }^{2}$ Las hemorroides se clasifican por su localización en: internas (por encima de la línea dentada y cubiertas por mucosa anal), externas (se originan por debajo de la línea dentada y cu-

Los autores declaran ausencia de conflictos de interés.

Homero Rodriguez-Zentner

drhomerorodriguez@gmail.com

Recibido: agosto de 2020. Aceptado: septiembre de 2020. biertas por anodermo) y mixtas. ${ }^{3} \mathrm{~A}$ su vez, las hemorroides internas se clasifican por el grado de prolapso. El interés práctico en la clasificación de las hemorroides, se basa en individualizar el tratamiento que más beneficiará al paciente, este puede ser conservador, instrumental o quirúrgico. ${ }^{4}$

Entre los tratamientos instrumentales ofrecidos, la ligadura con banda es el más utilizado, ya que tiene la menor tasa de recurrencia de síntomas y necesidad de retratamiento. ${ }^{5}$ Es relativamente seguro e indoloro. Se realiza de forma ambulatoria y consiste en colocar las bandas por arriba de la línea dentada, estrangulando la hemorroide, dejando una pequeña cantidad de tejido conectivo donde la cicatriz fije la mucosa a la submucosa y no se desarrolle 
nuevo tejido hemorroidal. ${ }^{6,7}$

Sin embargo, lograr la posición correcta de la banda en la base de la hemorroide o sobre el sitio de sangrado, lejos de la línea dentada, con el paciente despierto en la consulta, puede necesitar un alto grado de experticia y ser incómodo para el paciente. ${ }^{2}$

La ligadura con bandas, utilizando un endoscopio, fue descrita inicialmente para el tratamiento de las várices esofágicas por Stiegmann en 1989. ${ }^{8}$ Más de diez años después, el grupo de Trowers describe esta técnica para su uso en hemorroides, de forma reproducible y segura. Su estudio reclutó 20 pacientes, con un seguimiento de 5 meses, sin recurrencia en 19 (95\%) de los pacientes. ${ }^{9}$ En el mismo año Sadahiro describió que la observación del canal anal con el colonoscopio en retroflexión, facilitaba la identificación de hallazgos relacionados a sangrado y prolapso hemorroidal. ${ }^{10}$

En el presente estudio aportamos nuestra experiencia realizando este procedimiento con la ayuda visual del colonoscopio, utilizando el 6 Shooter $^{\circledR}$ Universal Saeed ${ }^{\circledR}$ Multi-Band Ligator y con el fin de analizar los beneficios de la ligadura endoscópica de hemorroides internas en pacientes con hemorroides internas grados I a III que no han respondido al tratamiento convencional.

\section{OBJETIVO}

Nuestro objetivo principal es analizar los beneficios de la ligadura endoscópica de hemorroides internas en pacientes con hemorroides internas grados I a III que no han respondido al tratamiento convencional, en un hospital privado en Ciudad de Panamá.

\section{MATERIAL Y MÉTODO}

Se revisaron observacional y retrospectivamente los expedientes clínicos de todos los pacientes con hemorroides sintomáticos, a los cuales se les realizó ligadura endoscópica entre febrero de 2015 y febrero de 2020 por el mismo grupo quirúrgico. El criterio de inclusión fue: pacientes con hemorroides sintomáticas entre los grados I a III sin respuesta a tratamiento convencional. Los criterios de exclusión fueron: presencia hemorroides grado IV, hemorroides complicadas con indicación de tratamiento quirúrgico y hemorroides grados I a III con componente externo. En todos los casos, el procedimiento se realizó en la suite de endoscopía, con el paciente sedado y monitorizado por un anestesiólogo. De los expedientes clínicos se recolectaron los datos sociodemográficos, comorbilidades, indicación para el procedimiento, tiempo de duración, tipo de ligadura empleada, dolor postprocedimiento (a las 24 horas, a la semana y a los 30 días), sangrado (a

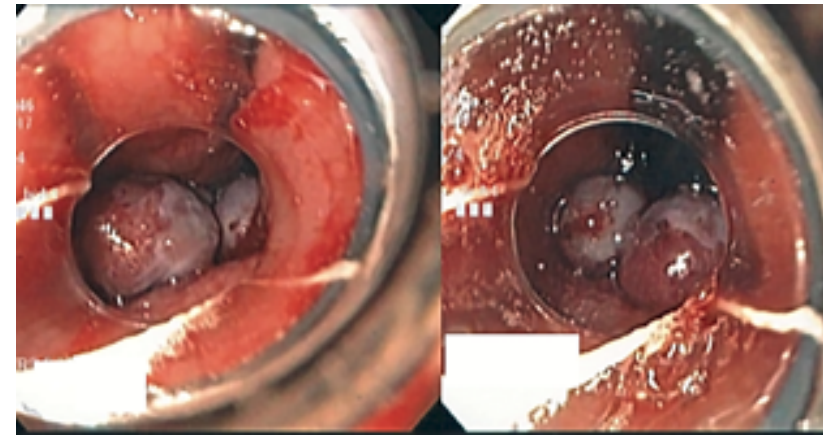

Figura 1: Ligadura endoscópica de hemorroides internas

TABLA 1: DATOS DEMOGRÁFICOS Y CLÍNICOS DE LOS PACIENTES ESTUDIADOS

$\begin{array}{ll}\text { Edad (años) } & \begin{array}{l}44,0 \text { años } \\ (21-78 \text { DE } 11,23)\end{array} \\ \begin{array}{ll}\text { Sexo } \\ \text { masculino } \\ \text { femenino }\end{array} & 92(63,0 \%) \\ \text { Indicación para el procedimiento } & 54(37,0) \\ \text { sangrado } & 106(72,5 \%) \\ \text { prolapso } & 11(7,5 \%) \\ \text { sangrado y prolapso } & 29(19,9 \%)\end{array}$

las 24 horas, a la semana y a los 30 días) y complicaciones.

\section{Análisis estadístico}

Para el análisis estadístico se utilizó SPSS (Statistical Package for Social Science) versión 21. Para la descripción de los datos se utilizó la media y desviación estándar (DE) para las variables cuantitativas y frecuencia y porcentaje para las variables cualitativas.

\section{RESULTADOS}

Se efectuó el procedimiento en 146 pacientes, 92 varones (63,01\%). La edad media de los pacientes fue 44,0 años (21-78 DE $\pm 11,23)$. El tiempo medio en el que se realizó el procedimiento fue 12,38 minutos (6-19 $\mathrm{DE} \pm 2,98)$.

La mayoría de las indicaciones fue dada por sangrado $72,6 \%$ (106), seguido por sangrado y prolapso $19,9 \%$ (29) y prolapso 7,5 \% (11) (Tabla 1).

En 120 pacientes se utilizaron ligaduras comunes, en 22 Wilson Cook, en 3 Smart Band, y a un paciente se le colocó Marflow AG (Figura 1).

A las 24 horas del procedimiento, según la Escala Verbal Numérica del Dolor (1-10), los pacientes describieron el dolor en una media de 4 , a los 7 días de 1,5 y a los 30 días de cero.

Después del procedimiento la mitad de pacientes presentó un sangrado leve durante 14 días, siendo el día de mayor sangrado el $7 \mathrm{mo}$. 
Todos los pacientes presentaron sensación de tenesmo al día siguiente del procedimiento. Siete días después del mismo, el 79 \% (63) aún lo presentaba. Treinta días después, solo tres pacientes continuaban con este síntoma (Tabla 2).

Dentro de las complicaciones evaluadas, 3 pacientes presentaron retención aguda de orina y 3 pacientes trombosis externa. Ningún paciente presentó fiebre, sepsis o perforación durante la retroflexión (Tabla 3).

\section{DISCUSIÓN}

Las hemorroides son la patología anorrectal más prevalente en el mundo occidental (un $4 \%$, aproximadamente) y son una causa muy frecuente de consulta por sangrado crónico. ${ }^{11}$ En pacientes con hemorroides grados I a III, se recomienda tratamiento conservador y procedimientos no quirúrgicos. ${ }^{12}$ Esta suele ser una patología de personas jóvenes. Al igual que en nuestro estudio, en la literatura la media de edad se sitúa en los 47 años de edad y afecta más al sexo masculino, lo cual también es consistente con nuestros resultados. ${ }^{13}$

El tratamiento no quirúrgico de las hemorroides, incluye la ligadura con banda, inyección de escleroterapia, coagulación infrarroja, criocirugía, hemorroidectomía por láser, entre otras. Estos tratamientos suelen realizarse de forma ambulatoria, sin anestesia. Así se reducen los costos relacionados a la estancia hospitalaria y al uso de quirófanos. ${ }^{14}$ Para nuestra práctica diaria y en vistas de la recuperación y el confort del paciente, este procedimiento lo realizamos con sedación y monitorización por un anestesiólogo. Todos estos procedimientos son ampliamente realizados en pacientes con estadios tempranos de hemorroides que no mejoran con tratamiento conservador. ${ }^{15}$ Lo que para algunos pacientes es muy importante es que con estas técnicas el tiempo de recuperación es mínimo o casi nulo, sin ausentismo laboral. En nuestra serie de pacientes, la media describió el dolor como 4 (en una escala del 1-10, siendo este último el máximo) a las 24 horas, lo cual permitía al día siguiente retomar las actividades cotidianas.

En la descripción de la técnica original, Barron propone tratar solamente un paquete hemorroides por sesión, sin embargo, muchos grupos reportan diferentes manejos. Ramzisham y Cazemier, en sus estudios randomizados controlados, reportan colocar entre 2 y 6 bandas por sesión. ${ }^{16,17}$

Akihisa en una serie de casos propone hasta un máximo de 8 bandas por sesión. ${ }^{18}$ En nuestra serie de pacientes, el máximo de bandas que colocamos por sesión fue de 3. Parece, en contraste a lo descrito originalmente, que se pueden colocar más de una banda para disminuir el nú-
TABLA 2: EVOLUCIÓN DURANTE EL PRIMER MES POST PROCEDIMIENTO

\begin{tabular}{llll} 
& 24 horas & 7 días & 30 días \\
\hline $\begin{array}{l}\text { Dolor } \\
\text { (EVN 1-10) }\end{array}$ & 4 & 2 & 0 \\
\hline $\begin{array}{l}\text { Sangrado (\%) } \\
143,1(98)\end{array}$ & $73(50)$ & $4,4(3)$ \\
\hline Tenesmo (\%) & $146(100)$ & $115.4(79)$ & $2.9(2)$
\end{tabular}

TABLA 3: COMPLICACIONES N (\%)

\begin{tabular}{ll} 
Complicaciones $n(\%)$ & \\
\hline Retención aguda de orina & $3(2,05)$ \\
\hline Trombosis externa & $3(2,05)$ \\
\hline Fiebre & 0 \\
Sepsis & 0 \\
\hline Perforación & 0
\end{tabular}

mero de sesiones necesarias, sin embargo, no se ha llegado a un consenso del número máximo de bandas por sesión permitido.

Tradicionalmente, la ligadura con bandas se realiza con un proctoscopio rígido el cual, comparado con el colonoscopio, es más difícil de maniobrar y aporta una visión más limitada que este último. Nosotros preferimos el uso del colonoscopio ya que permite además de la visión anterógrada, una visión en retroflexión, lo cual proporciona información importante de la presentación clínica y permite documentar hallazgos. ${ }^{19}$

En la literatura publicada, la tasa de complicaciones se mantiene entre 4 a $10 \%$ (principalmente dolor y sangrado), las complicaciones fatales o decesos son inusuales. En nuestro estudio, la tasa de complicaciones se situó en 7,5\%, las mismas fueron menores y se manejaron de forma ambulatoria. Resultados similares se publicaron en una de las series más grandes reportadas hasta ahora con la técnica (576 pacientes) de un grupo en Taiwán. ${ }^{20}$

En un estudio prospectivo randomizado entre ligadura con bandas convencional y ligadura con el uso de colonoscopio, realizado por Wehermann et al., se observó que los pacientes en los cuales se realizó el procedimiento con ayuda del endoscopio necesitaron menor número de sesiones $1,8[0,8]$ vs. $2,4[0,9]$ para control de la enfermedad. ${ }^{21}$ Una limitante en nuestro estudio es que, al realizar todas las colocaciones de las bandas con ayuda del endoscopio, no tenemos una serie de paciente similar: los beneficios del uso del colonoscopio son teóricos. En el estudio mencionado anteriormente, ellos observaron que ambas técnicas, la convencional y endoscópica, aportaban los mismos resultados en la intención a tratar, control del sangrado hemorroidal y similar tasa de complicaciones, lo cual es esperado ya que las dos técnicas están basadas en el mismo mecanismo de acción. ${ }^{21}$ 


\section{CONCLUSIONES}

La ligadura endoscópica de hemorroides es una técnica reproducible, segura, que permite el tratamiento de hemorroides sintomáticas grados I a III, en su gran mayoría, en una sola sesión.

\section{REFERENCIAS}

1. Barleben A, Mills S. Anorectal anatomy and physiology. Surg Clin North Am 2010;90:1-15.

2. Sneider EB, Maykel JA. Diagnosis and management of symptomatic hemorrhoids. Surg Clin North Am 2010;90:17-32.

3. Lohsiriwat V. Hemorrhoids: from basic pathophysiology to clinical management. World J Gastroenterol 2012;18:2009-17.

4. Lohsiriwat V. Treatment of hemorrhoids: A coloproctologist's view. World J Gastroenterol 2015;21:9245-52.

5. Lohsiriwat V. Approach to hemorrhoids. Curr Gastroenterol Rep 2013;15:332.

6. Blaisdell PC. Office ligation of internal hemorrhoids. Am J Surg 1958;96:401-4.

7. Barron J. Office ligation of internal hemorrhoids. Am J Surg 1963;105:563-70.

8. Stiegmann GV, Goff JS, Sun JH, et al. Endoscopic variceal ligation: an alternative to sclerotherapy. Gastrointest Endosc 1989;35:431-34.

9. Trowers EA, Ganga U, Rizk R, et al. Endoscopic hemorrhoidal ligation: preliminary clinical experience. Gastrointest Endosc 1998;48:49-52.

10. Sadahiro S, Mukai M, Tokunaga N, et al. A new method of evaluating hemorrhoids with the retroflexed fiberoptic colonoscope. Gastrointest Endosc 1998;48:272-75.

11. Berkelhammer C, Moosvi SB. Retroflexed endoscopic band ligation of bleeding internal hemorrhoids. Gastrointest Endosc 2002;55:532-37.

12. Riss S, Weiser FA, Schwameis K, et al. Haemorrhoids, constipation and faecal incontinence: is there any relationship? Colorectal Dis

\section{1;13:227-33}

13. Arora G, Mannalithara A, Mithal A, Triadafilopoulos G, Singh G. Concurrent conditions in patients with chronic constipation: A population-based study. PLoS One 2012;7:e42910.

14. Cocorullo G, Tutino R, Falco N, et al. The non-surgical management for hemorrhoidal disease. A systematic review. G Chir 2017;38:5-14.

15. François P, Laurent S, François A. Risk factors associated with hemorrhoidal symptoms in specialized consultation. Gastroenterol Clin Biol 2005;29:1270-74.

16. Cazemier M, Felt-Bersma RJ, Cuesta MA, Mulder CJ. Elastic band ligation of hemorrhoids: Flexible gastroscope or rigid proctoscope? World J Gastroenterol 2007;13:585-87.

17. Ramzisham AM, Sagap I, Nadeson S, Ali IM, Hasni MJ Prospective randomized clinical trial on suction elastic band ligator versus forceps ligator in the treatment of haemorrhoids. Asian J Surg 2005;28:241-45.

18. Akihisa F, Toru K, Hiroaki A, et al. Retroflexed endoscopic multiple band ligation of symp- tomatic internal hemorrhoids. Gastrointest Endosc 2004;59:380-84.

19. Wehrmann T, Riphaus A, Feinstein J, et al. Hemorrhoidal elastic band ligation with flexible videoendoscopes: a prospective, randomized comparison with the conventional technique that uses rigid proctoscopes. Gastrointest Endosc 2004;60:191-15.

20. Su MY, Chiu CT, Wu CS, et al. Endoscopic hemorrhoidal ligation of symptomatic internal hemorrhoids. Gastrointest Endosc 2003;58:871-74.

\section{COMENTARIO}

Los autores presentan una experiencia de 145 pacientes con hemorroides internas tratados con ligaduras endoscópicas, con excelentes resultados a corto plazo y baja morbilidad. La población tratada es joven (promedio 44 años) con hemorroides grados I a III (no se menciona el detalle de cada subgrupo), con un índice de indicación por prolapso de solo un $27 \%$. Hubiera sido interesante evaluar los resultados por grado, dado que también incluyen el Grado I, sabiendo que las recomendaciones actuales no suelen incluir la ligadura convencional en este grupo.

Se destaca que las complicaciones son leves y comunes a las demás técnicas y factibles de tratamiento ambulatorio sin secuelas.

Como técnica per se es prometedora cuando es realizada por cirujanos endoscopistas como los autores, y a diferencia de la ligadura convencional requiere de sedación por la realización de una colonoscopía asociada, y sumaria un costo, teóricamente, mayor tanto por el uso del endoscopio como por las bandas endoscópicas en sí al ser comparadas con las convencionales. Como enuncian los autores sería necesario realizar nuevas investigaciones, tanto prospectivas como comparativas en orden de analizar recurrencia y resultados a largo plazo.

Hugo Amarillo

Sanatorio Modelo. San Miguel de Tucumán, Tucumán. Argentina. 